\title{
Successful Medical and Surgical Management of Massive Hemoperitoneum in Early Pregnancy
}

\section{Giovanna Rosica, Daniela Bucari, Giovanni Pastore, Angela Musella*, Maria Pia Giorno, Valeria Ebano and Vincenzo Spina}

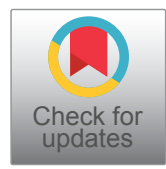

Department of Obstetrics and Gynecology, San Camillo de Lellis Hospital, Viale Kennedy, Italy

*Corresponding author: Angela Musella, Department of Obstetrics and Gynecology, San Camillo de Lellis Hospital, Viale Kennedy, 02100, Rieti, Italy

\begin{abstract}
Introduction: There are several cases reported in literature about management of adnexal masses and hemoperitoneum during pregnancy, however there is still no clear and standardized treatment. Through this manuscript we want to report our experience about the successfully treatment of massive hemoperitoneum due to corpus luteum rupture during first trimester of pregnancy.

Case description: A Caucasian young patient, pregnant at early gestational age, referred to our Department because of acute pelvic and abdominal pain due to rupture of left ovary corpus luteum. After conservative approach with medical treatment and strict observation a surgical intervention became necessary because of patient's conditions worsening. A left adnexectomy under minilaparotomic approach was performed, without complications. After that, the pregnancy went on uneventfully, with adequate medical treatment and periodic controls, until the patient gave birth to a healthy baby.
\end{abstract}

Conclusions: An adequate and careful treatment of promptly diagnosed gynecological emergencies occurring during gestational period is feasible without invalidate pregnancy outcome.

\section{Introduction}

Corpus luteum is characterized by four developing stages: proliferation, vascularization, maturation and regression. During pregnancy, it develops after oocyte fertilization under corionic gonadotropin (BhCG) stimulation extending on most of the ovarian surface. The size and structure of corpus luteum may be different reaching the maximum size at 7-9 weeks of gestation. At the end of pregnancy, it is usually no more detectable.
In case of acute pelvic pain during pregnancy, differential diagnosis should be done with other conditions such as ectopic pregnancy, endometriosis, dermoyd ovarian cysts or neoplasia. According to a recent study, adnexal masses are discovered in 1 per $76-1$ per 2328 deliveries [1]. The incidence of an adnexal cancer in pregnancy is one per $12,000-47,000$. It is, therefore, considered the second most common gynecological mass detected in pregnancy following benign cysts [2].

The course of adnexal masses during pregnancy is usually spontaneous resolution of simple anechoic cysts less than $5 \mathrm{~cm}$ in diameter [3]. It has been reported that pregnant patients have a $1 \%$ increased risk of ovarian torsion compared to non-pregnant patients. Most of the ovarian torsion cases occur in the first trimester; thereafter, the risk of torsion decreases $[3,4]$.

However, high risk of severe complications such as miscarriage, premature amniotic rupture, premature labor and delivery is reported. In case of cyst size higher than $5 \mathrm{~cm}$ treatment options include: Wait and see or surgical management. Time to surgical removal of cyst is until 28 gestational weeks because of increased risk of premature labor, however the optimal timing for surgery is during the second trimester [1]. Nevertheless, whenever a malignancy is suspected, surgery should be performed at any time of pregnancy, through a laparoscopic approach if possible. Corpus luteum ruptures may occur spontaneously, requiring differential diagnosis with other conditions and prompt detection of emergency symptoms. There are several cases report-

Citation: Rosica G, Bucari D, Pastore G, Musella A, Giorno MP, et al. (2021) Successful Medical and Surgical Management of Massive Hemoperitoneum in Early Pregnancy. Clin Med Rev Case Rep 7:334. doi.org/10.23937/2378-3656/1410334

Accepted: January 07, 2021: Published: January 09, 2021

Copyright: (C) 2021 Rosica G, et al. This is an open-access article distributed under the terms of the Creative Commons Attribution License, which permits unrestricted use, distribution, and reproduction in any medium, provided the original author and source are credited. 


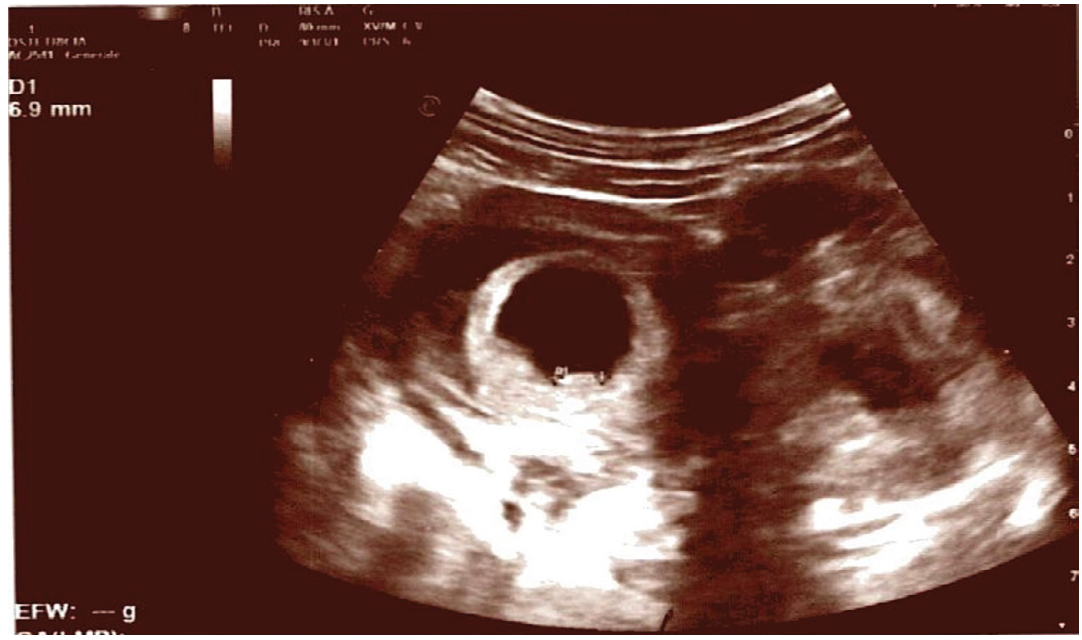

Figure 1: Intrauterine gestationalsac with single embryo (CRL: $6.9 \mathrm{~mm}$ ) endowed with cardiac activity. No free pelvicfluid.

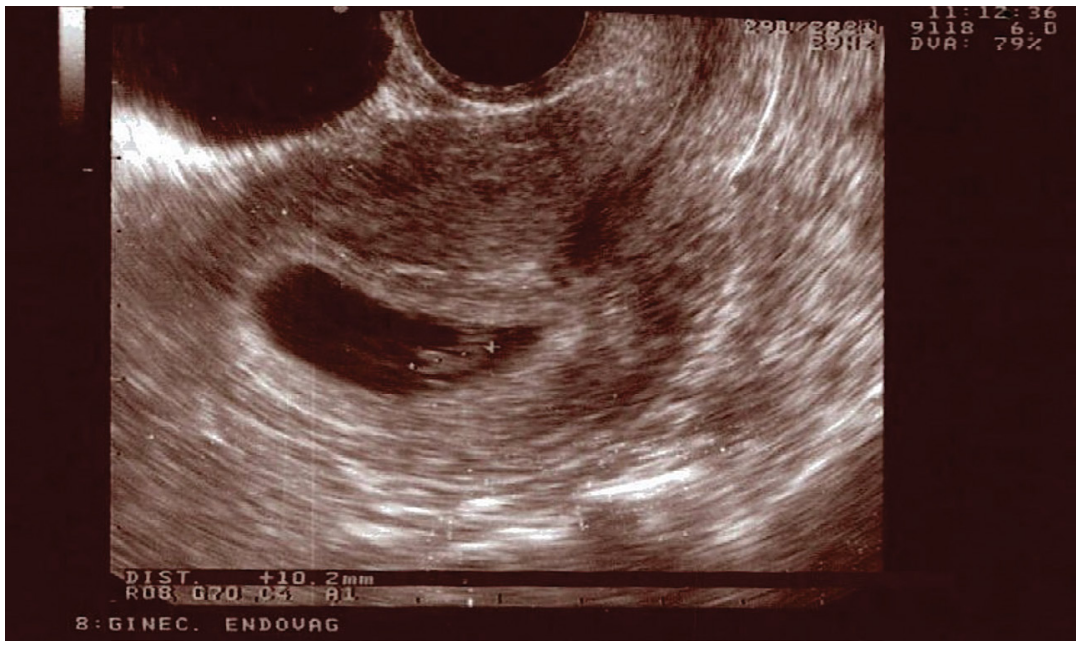

Figure 2: Intrauterine gestationalsac with single embryo (CRL: $10 \mathrm{~mm}$ ) endowed with cardiac activity. No free pelvicfluid.

ed in literature about management of adnexal masses and hemoperitoneum during pregnancy [5-8], however there is still no clear and standardized treatment. Through this manuscript, we want to report our favorable experience with medical and surgical treatment of massive hemoperitoneum due to corpus luteum rupture during first trimester of pregnancy.

\section{Case Description}

A Caucasian 34-years-old patient, referred to our Department of Obstetrics and Gynecology at 6 weeks of gestational age complaining pelvic pain and vaginal bleeding. She had a history of previous surgery: A uterine curettage for miscarriage 5 years before, a right salpingectomy for ectopic pregnancy and appendectomy. The patient obtained the current pregnancy thorough in vitro fertilization with embryo transfer. The clinical and obstetrical evaluation at time of admission revealed no abdominal or pelvic pain, scarce vaginal bleeding. Laboratory exams were normal at admission with a regular increase of corionic gonadotropin dosage (Hgb $11.6 \mathrm{~g} /$ dl; WBC 8,2; PLT 301.000; ßhCG: 11.200 mUl/ml). Pel- vic and transvaginal ultrasound revealed regular intrauterine gestational sac with single embryo of $6.9 \mathrm{~mm}$ in crown-rump lenght (CRL) and presence of cardiac activity, corresponding to 6 weeks course of gestational age (Figure 1), with left ovary increased in size. The patient was hospitalized and she started medical treatment with progesteron, cortisons, antispasmodics and antibiotics because of vaginal infection revealed through vaginal swabs. Blood exams performed during hospitalization were within the normal ranges; the patient was always apyretic and a subsequent ultrasound evaluation, some days after, disclosed intrauterine gestational sac with one embryo of $10.2 \mathrm{~mm}$ in crown-rump lenght and presence of cardiac activity (Figure 2). Due to the improvement of patient symptoms, she was discharged with prescription of medical therapy with progesteron and antispasmoidcs and strictserial controls.

After three days from discharge, the patient came back to the hospital complaining severe pelvic pain with back irradiation. Blood exams revealed a mild anemia (Hgb level $10 \mathrm{~g} / \mathrm{dl}$ ) with leucocytosis (WBC: 13.5), vital parameters were normal. Trans-abdominal and trans- 


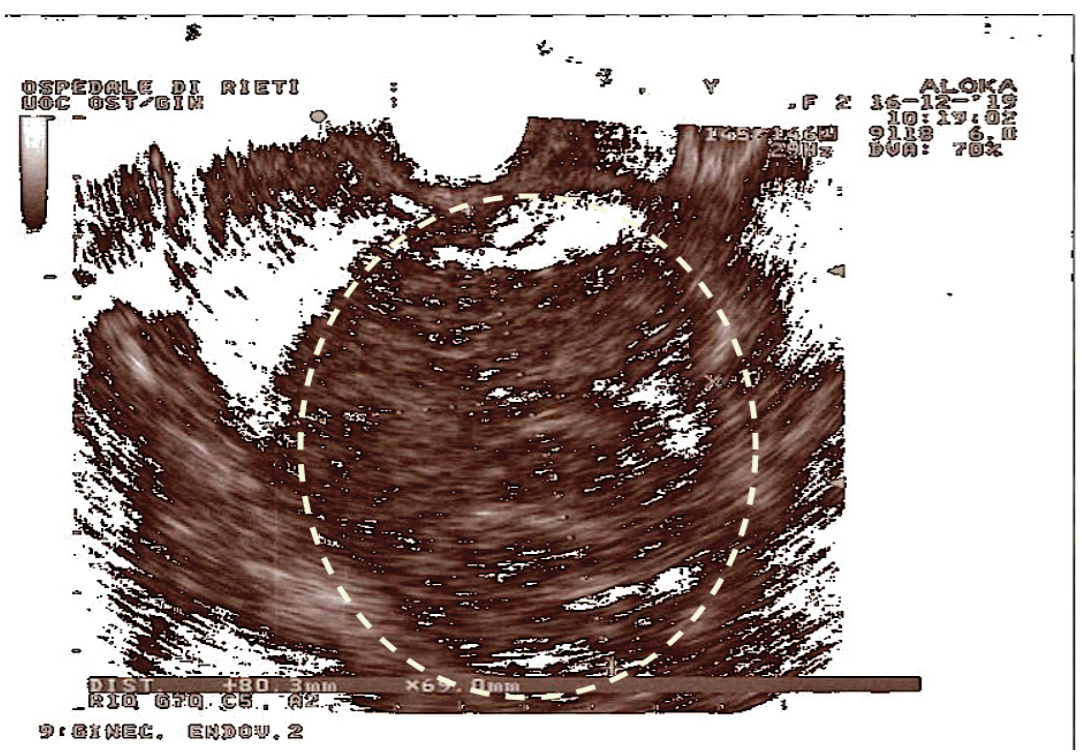

Figure 3: Pelvic mass on the left pelvic side measuring, without evidence of free pelvic fluid.

vaginal pelvic ultrasound disclosed the presence of intrauterine gestational sac with one embryo with cardiac activity and the presence of a pelvic mass on the left pelvic side measuring $80 \times 69 \mathrm{~mm}$ in diameters, without blood flow at power Doppler, and without free pelvic fluid (Figure 3). Thus, considering the stable patient's general conditions and the absence of free fluid in abdominal cavity, she started strict controls through laboratory exams, pelvic ultrasound and treatment with antispasmoidcs drugs, with improvement of symptoms.

The day after, the patient presented a sudden worsening of symptoms, blood exams revealed a severe anemia ( $\mathrm{Hgb}$ level $7 \mathrm{~g} / \mathrm{dl}$ ), with hypothension and lipotimic signs. Abdominal evaluation was different from before, with severe pain at abdominal palpation and high positive Blumberg sign. Pelvic ultrasound evaluation revealed a painful left side pelvic mass, with vascular ring suggestive of hematoma, mild hemoperitoneum, and vital embryo within the uterine cavity. Thus, after adequate informed consent was obtained, surgery was promptly decided. A minilaparotomic approach was used because of the unstable conditions of the patient: Bloody corpus luteum, a large left side pelvic mass with clots and fibrin adhered to the left ovary, a left twisted tuba full of clots and hemoperitoneum were found at surgery. The pregnant uterus appeared normal.

Accurate abdominal and pelvic toilette with aspiration of approximately one liter of blood and clots from the abdominal cavity, and left adnexectomy because of bad conditions of the adnexum were performed. The post-operative course was uneventful, but transfusion of two blood bags was needed. Histology revealed a bloody corpus luteum with para-ovaric cyst and a Walthard nest.

The patient was discharged on post-operative day 4 , with prescription of estrogenic and progestinic treatment and strict serial controls.
The subsequent pregnancy course was uneventful. The patient underwent cesarean section, upon her request, at 38 gestational weeks: A female baby, Apgar 9/10, weight $2800 \mathrm{gr}$, was delivered. The post-operative course was uneventful and the patient and her baby were discharged on postoperative day 3 .

\section{Discussion}

Spontaneous hemoperitoneum may result from several gynecological and obstetrical conditions. Corpus luteum rupture during pregnancy is rare throughout the first trimester. Symptoms are not specific and differential diagnosis with other pathological conditions during pregnancy is needed. Diagnosis of corpus luteum rupture is based on patient's general conditions, blood exams, and above all pelvic and transvaginal ultrasound: Usually, a mass on the pelvic side with uneven appearance and intralesional bleedingis detected [9]. Magnetic resonance imaging (MRI) evaluation is important to have a better visualization of posterior and lateral pelvic regions that could be not easily detectable during pregnancy with ultrasound. Conservative management of adnexal masses during pregnancy is possible in stable patients or with no severe symptoms, otherwise a surgical approach is needed. Frequently, signs of abdominal resentment are highly severe in case of corpus luteum rupture and hemoperitoneum, as in our case. Conservative surgical treatment should be performed whenever possible, in order to preserve future fertility of patients [10]. There are few reports of ruptured corpus luteum in pregnancy with hemoperitoneum requiring emergency treatment [11-13].

Takeda, et al. reported on a 15-year-old girl, vitally at risk because of intra-abdominal bleeding. Transvaginal ultrasound revealed intrauterine gestation and fluid in the abdomen, thus urgent laparoscopy was performed, showing bleeding from the corpus luteum cyst and hemostasis was achieved. The intrauterine pregnancy 
was electively terminated because the pregnancy was not desired [11]. Vidaković, et al. reported a case of a 35-year-old patient admitted to the hospital because of severe abdominal pain with positive BhCG and no intrauterine gestational sac visualized on ultrasonography. Because of patient's unstable conditions, urgent laparoscopy was performed and ruptured luteal cyst was found and removed. Postoperatively, normal intrauterine gestation was visualized [12].

Moreover, Bauman, et al. in 2018 reported about a case of ruptured corpus luteum during first trimester of pregnancy in a 33-years-old woman. In this case, the patient presented to the hospital because of lower abdominal pain of sudden onset. Transvaginal ultrasound showed hyperechogenic endometrium, with no gestational sac and a large amount of fluid in the pouch of Douglas, corpus luteum was seen in the left ovary, surrounded by inhomogeneous echoes of coagula. The patient was successful treated with laparoscopy and after that, the pregnancy was uneventful with a spontaneous vaginal delivery of a healthy baby at 41 weeks of gestation [13]. In our case, there was a large involvement of ovarian and tubal tissues, thus it was not possible to preserve the left adnex.

\section{Conclusions}

In conclusion, it is well known that pregnancy represents a very delicate physiological condition in which many balances are upset and any event could be harmful for gestational outcome. Management of gynecological emergencies during pregnancy is not so clear or standardized. Although extremely rare, rupture of corpus luteum in early pregnancy with hemoperitoneum can cause acute abdomen and should be considered in diagnostic process. However, because of acute symptoms, therapy is mainly surgical with a laparoscopy as gold standard, if possible. Our experience suggests that a favorable outcome of pregnancy is possible through adequate treatment of these pathological conditions.

\section{Conflict of Interest Statement}

All authors declare they have no conflict of interest.

\section{Statement of Equal Authors' Contribution}

All authors contribute equally to the drafting, writing and revision of the manuscript.

\section{References}

1. Aggarwal $P$, Kehoe $S$ (2011) Ovarian tumours in pregnancy: a literature review. Eur J Obstet Gynecol Reprod Biol 155: 119-124.

2. Garrett WJ, Coppleson M, Mclnerney RJ (1990) Ultrasound and ovarian cysts. Med J Aust 152: 52.

3. Yacobozzi M, Nguyen D, Rakita D (2012) Adnexal masses in pregnancy. Semin Ultrasound CT MR 33: 55-64.

4. Schwartz N, Timor-Tritsch IE, Wang E (2009) Adnexal masses in pregnancy. Clin Obstet Gynecol 52: 570-585.

5. Kayaba H, Tamura H, Shirayama K, Murata J, Fujiwara $Y$ (1996) Hemorrhagic ovarian cyst in childhood: a case report. Journal of Pediatric Surgery 7: 978-979.

6. Roche O, Chavan N, Aquilina J, Rockall A (2012) Radiological appearances of gynaecological emergencies. Insights into Imaging 3: 265-275.

7. Coulier B, Malbecq S, Brinon PE, Ramboux A (2008) MDCT diagnosis of ruptured tubal pregnancy with massive hemoperitoneum. Emergency Radiology 15: 179-182.

8. Fiaschetti V, Ricci A, Scarano AL, Liberto V, Citraro D, et al. (2014) Hemoperitoneum from corpus Luteal Cyst Rupture: A Practical Approach in Emergency Room. Case Report in Emergency Medicine 2014: 252657.

9. Bromley B, Benacerraf B (1997) Adnexal masses during pregnancy accuracy of sonographic diagnosis and outcome. J Ultrasound M 16: 447-452.

10. Oelsner G, Cohen SB, Soriano D, Admon D, Maschiach $S$, et al. (2003) Minimal surgery for the twisted ischaemic adnexa can preserve ovarian function. Human Reprod 18: 2599-2602.

11. Takeda A, Sakai K, Mitsui T, Nakamura H (2007) Management of ruptured corpus luteum cyst of pregnancy occurring in a 15-year-old-girl by laparoscopic surgery with intraoperative autologous blood transfusion. J Pediatr Adolesc Gynecol 20: 97-100.

12. Vidakovic S, Dokic M, Vilendecic Z, Djaconovic Maravic M (2013) Ruptured Corpus luteum Cyst In Early Pregnancy. A case Report. Srp ArhLek 141: 689-692.

13. Bauman R, Horvat G (2018) Management of ruptured corpus luteum with hemoperitoneum in early pregnancy. A case report. Acta Clin Croat 57: 785-788. 